\title{
Estudo da excreção urinária de cálcio, potássio e sódio com o emprego de citrato de potássio na hipercalciúria idiopática na criança
}

\author{
Study of urinary excretion of calcium, potassium and sodium using potassium citrate in children with \\ idiopathic hypercalciuria
}

Amélia Arcângela T. Trindade ${ }^{1}$, Célia Sperandeo Macedo², Márcia Camegaçava Riyuzo ${ }^{3}$, Carlos Roberto Padovani', Herculano Dias Bastos ${ }^{5}$

\section{RESUMO}

Objetivo: Estudar as relações entre a excreção urinária de cálcio, sódio e potássio e a associação sódio/potássio urinários em crianças com hipercalciúria idiopática em dieta habitual, antes e depois da administração de citrato de potássio na dose de $1 \mathrm{mEq} / \mathrm{kg} / \mathrm{dia}$.

Métodos: Foram estudadas prospectivamente 26 crianças: $19(73 \%)$ meninos e sete (27\%) meninas com idade entre dois e 13 anos, portadores de hipercalciúria idiopática recémdiagnosticada por dosagem de cálcio em urina de 24 horas $\geq 4 \mathrm{mg} / \mathrm{kg} /$ dia. O citrato de potássio foi administrado na dose de $1 \mathrm{mEq} / \mathrm{kg} / \mathrm{dia}$. Foram realizadas dosagens séricas e em urina de 24 horas de cálcio $(\mathrm{Ca})$, potássio $(\mathrm{K})$, sódio $(\mathrm{Na})$ e creatinina $(\mathrm{Cr})$, antes e 15 dias depois da administração diária do citrato de potássio. Para comparar os resultados de cálcio/creatinina $(\mathrm{Ca} / \mathrm{Cr})$, potássio/creatinina $(\mathrm{K} / \mathrm{Cr})$ e sódio/potássio $(\mathrm{Na} / \mathrm{K})$ urinários nos dois momentos, aplicou-se o teste não-paramétrico de Wilcoxon. Para a análise das associações entre $\mathrm{Ca} / \mathrm{Cr}$ e $\mathrm{K} / \mathrm{Cr}$ e entre $\mathrm{Ca} / \mathrm{Cr}$ e $\mathrm{Na} / \mathrm{Cr}$ foi utilizado o coeficiente de correlação de Pearson. Considerou-se significante $p<0,05$.

Resultados: Após o uso de citrato de potássio, ocorreu significativa redução da calciúria e da relação $\mathrm{Na} / \mathrm{K}$ urinários, bem como elevação na caliúria. Não houve modificação da excreção urinária de sódio.

Conclusões: Em dieta habitual, o citrato de potássio eleva a caliúria e diminui a calciúria em criança hipercalciúricas, sendo um eficaz recurso terapêutico.

Palavras-chave: cálcio; criança; adolescente; citrato de potássio.

\section{ABSTRACT}

Objective: Evaluate the relationships among the urinary excretion of calcium (UCa), potassium (UK), sodium (UNa) and the ratio between $\mathrm{UNa} / \mathrm{UK}$ in children with idiopathic hypercalciuria and a regular diet, before and after $1 \mathrm{mEq} / \mathrm{kg} /$ day potassium citrate administration.

Methods: 26 children with idiopathic hypercalciuria (UCa $\geq 4 \mathrm{mg} / \mathrm{kg} /$ day) were prospectively studied: 19 (73\%) boys and seven (27\%) girls between two and 13 years old. Potassium citrate was administered: $1 \mathrm{mEq} / \mathrm{Kg} /$ day twice a day for 15 days. Blood and 24-hour urinary determinations of calcium, potassium, sodium and creatinine were done in two periods: before and after the 15-day administration of potassium citrate. The following urinary ratios were analyzed before and after potassium citrate use by Wilcoxon test: calcium/creatinine (UCa/UCr), potassium/creatinine (UK/UCr) and sodium/creatinine (UNa/UCr). The association between $\mathrm{UCa} / \mathrm{UCr}, \mathrm{UK} / \mathrm{UCr}$ and $\mathrm{Ca} / \mathrm{Cr}, \mathrm{UCa} / \mathrm{UCr}$ and $\mathrm{UNa} / \mathrm{UK}$ were analyzed by Pearson's correlation. Significance was considered for $p<0.05$.

Results: After potassium citrate use, there were significant reductions of $\mathrm{UCa}$ and $\mathrm{UNa} / \mathrm{UK}$ ratios, as well as a significant increase of UK. The UNa did not change.

Conclusions: Children with idiopathic hypercalciuria and regular diet treated with daily potassium citrate increased their potassium urinary excretion and decreased their calciuria.

Key-words: calcium; child; adolescent; potassium citrate.

Endereço para correspondência:

Amélia Arcângela T. Trindade

Hospital das Clínicas da Faculdade de Medicina Unesp - Botucatu,

Departamento de Pediatria

Distrito de Rubião Junior, s/n

CEP 18000-000 - Botucatu/SP

E-mail: ametrindade@uol.com.br

Recebido em: 11/9/2006

Aprovado em: 17/4/2007 


\section{Introdução}

A hipercalciúria idiopática é uma alteração metabólica caracterizada pela excreção urinária aumentada de cálcio em pacientes normocalcêmicos. Na infância, a excreção é considerada aumentada quando o cálcio urinário é maior ou igual a $4 \mathrm{mg} / \mathrm{kg}$ em 24 horas, em pelo menos uma de três amostras colhidas em dias não consecutivos. Outro parâmetro de diagnóstico é a relação cálcio/creatinina $(\mathrm{Ca} / \mathrm{Cr})$ maior que 0,21 em amostra de urina matinal e em jejum ${ }^{(1-3)}$.

Hematúria macro e microscópica e/ou dor abdominal são achados clínicos comuns descritos em pacientes hipercalciúricos $^{(4,5)}$. Entretanto, outros sinais e sintomas como disúria, urgência, incontinência urinária, dor suprapúbica ou uretral e, às vezes, infecção urinária sugerem a pesquisa de hipercalciúria idiopática ${ }^{(2)}$. A hipercalciúria idiopática é freqüentemente encontrada na nefrolitíase, acometendo, em média, $50 \%$ dos pacientes pediátricos litiásicos ${ }^{(2,6,7)}$.

A etiologia ainda permanece controversa. Dentre os vários mecanismos fisiopatogênicos propostos, destacam-se: aumento primário da absorção intestinal de cálcio, redução da reabsorção tubular renal de cálcio, perda renal primária de fosfato, aumento primário da reabsorção óssea, aumento primário na síntese de 1,25 diidroxivitamina $\mathrm{D}_{3}$, aumento da produção renal de prostaglandinas $\mathrm{E}_{2}$ e distúrbios tubulares renais associados ${ }^{(2,3,8,9)}$. Atualmente, admite-se que mais de um mecanismo ocorra no mesmo paciente e que haja interligação entre eles.

A influência de íons na excreção urinária de cálcio tem sido largamente estudada ${ }^{(10-15)}$. Lemann et al, em $1991^{(16)}$, demonstraram existir relação entre ingestão de potássio e excreção renal de cálcio em adultos normais, verificando que a alta ingestão de potássio resultava em diminuição da excreção renal de cálcio. Em crianças com hipercalciúria idiopática, observou-se que a excreção renal de potássio era menor do que a observada em crianças normais, após sobrecarga oral de cálcio. Além disso, o aumento da calciúria era acompanhado por significante queda da concentração tubular renal de potássio, sugerindo que o aumento na concentração luminal renal de cálcio iniba a secreção renal de potássio ${ }^{(17)}$. Osório et $a l^{(18)}$ demonstraram existir relação inversa entre a excreção renal de potássio e cálcio e notaram o efeito hipocalciúrico da administração de potássio em crianças com hipercalciúria idiopática. Estes autores verificaram que a redução de ingestão de sódio influi no grau da calciúria. Outros estudos evidenciaram diminuição da gravidade clínica da litíase urinária em crianças hipercalciúricas, obtida com menor ingestão de sódio ${ }^{(4,14)}$.
O citrato de potássio tem sido amplamente utilizado no tratamento da litíase cálcica ${ }^{(19)}$ e, como medicamento de primeira escolha, em crianças com hipercalciúria idiopática ${ }^{(2)}$. Estudo mostrou que o aumento na excreção de potássio eleva a reabsorção tubular renal de cálcio, sendo também atribuída ao citrato de potássio esta propriedade terapêutica ${ }^{(16)}$. Neste contexto, o objetivo deste trabalho é estudar a excreção renal de potássio, cálcio e sódio em crianças hipercalciúricas, antes e após o emprego oral de citrato de potássio.

\section{Métodos}

No período de um ano, 36 crianças iniciaram o estudo; destas, dez foram excluídas pelos seguintes motivos: não realização de coleta urinária e sangüínea antes ou após o tratamento; não adesão ao uso regular da medicação, observada pelo número de cápsulas trazidas na consulta após 15 dias; e ausência de consentimento familiar.

Assim trata-se de um estudo prospectivo de 26 crianças: $19(73 \%)$ meninos e sete (27\%) meninas, com idade entre dois e 13 anos, portadoras de hipercalciúria idiopática e acompanhadas pelo Ambulatório de Nefrologia Pediátrica do Hospital das Clínicas da Faculdade de Medicina de Botucatu da Universidade Estadual Paulista (Unesp), no período de um ano. Este estudo foi iniciado após sua aprovação pelo Comitê de Ética em Pesquisa da instituição.

Depois de assinado o consentimento livre, esclarecido por um dos responsáveis, foi preenchido um protocolo contendo informações do paciente sobre procedência e sintomatologia inicial, sendo então solicitada e orientada a coleta de urina de 24 horas e de sangue para dosagens de cálcio, sódio, potássio e creatinina, antes e 15 dias após o uso de citrato de potássio.

O citrato de potássio foi ministrado na dose de $60 \mathrm{mg} / \mathrm{kg} / \mathrm{dia}$ ou $1 \mathrm{mEq} / \mathrm{kg} /$ dia em duas tomadas, fornecido em forma de cápsula de 500mg, em quantidade suficiente para completar 15 dias de tratamento. O paciente e os familiares eram orientados a manter a dieta habitual. Depois de 15 dias do uso da medicação, foi colhida nova amostra de urina de 24 horas e sangue para dosagem de cálcio, sódio, potássio e creatinina.

$\mathrm{Na}$ análise estatística dos resultados, utilizou-se o teste não-paramétrico de Wilcoxon para as amostras dependentes sangüíneas e urinárias: creatinina $(\mathrm{Cr})$, cálcio $(\mathrm{Ca})$, sódio $(\mathrm{Na})$, potássio $(\mathrm{K})$, e também das relações $\mathrm{Ca} / \mathrm{Cr}, \mathrm{Na} / \mathrm{Cr}, \mathrm{K} /$ $\mathrm{Cr}, \mathrm{Na} / \mathrm{K}$, comparando os momentos pré e pós-tratamento. Para medir as associações $(\mathrm{Ca} / \mathrm{Cr}$ e $\mathrm{K} / \mathrm{Cr}, \mathrm{Ca} / \mathrm{Cr}$ e Na/Cr) nos momentos pré e pós-tratamento, foi utilizado o coeficiente 
de correlação de Pearson. Em toda a análise, adotou-se nível de significância de $5 \%$ para rejeição da hipótese de nulidade e considerou-se $p<0,05$ como significante ${ }^{(20)}$.

\section{Resultados}

$\mathrm{Na}$ Tabela 1, encontram-se os dados referentes à caracterização das crianças quanto à idade e ao gênero. Chama atenção a prevalência maior em meninos, entre oito e 13 anos. Também foi diagnosticada hipercalciúria idiopática na faixa etária entre um e quatro anos.

As principais queixas apresentadas pelos pacientes no início do quadro estão demonstradas no Gráfico 1: hematúria, dor lombar ou abdominal, disúria e infecção urinária (ITU).

As dosagens séricas de cálcio, sódio, potássio e creatinina antes e após emprego de citrato de potássio não demonstraram variação estatisticamente significativa (Tabela 2).

Com relação às dosagens urinárias, observou-se haver diminuição significativa da excreção urinária de cálcio e aumento significativo na excreção urinária de potássio após uso do citrato de potássio, quando se comparou isoladamente os dois momentos para cada eletrólito. Com relação ao sódio, não houve variação estatística, quando se comparou de forma isolada os dois momentos do estudo (Tabela 2).

Ao comparar a relação cálcio/creatinina $(\mathrm{Ca} / \mathrm{Cr})$ urinários nos dois momentos, notou-se variação estatisticamente significativa, com diminuição desta relação no momento pós-citrato $(p<0,05)$. Houve aumento significativo da relação potássio/creatinina $(\mathrm{K} / \mathrm{Cr})$ urinários no momento pós, quando comparado ao momento pré-uso do citrato de potássio $(p<0,01)$. Quanto à relação sódio/creatinina $(\mathrm{Na} / \mathrm{Cr})$ urinária nos dois momentos, não houve variação estatística (Tabela 2).

A relação sódio e potássio $(\mathrm{Na} / \mathrm{K})$ urinários nos dois momentos estudados diminuiu significativamente, como mostra a Tabela 2.

Tabela 1 - Distribuição dos pacientes de acordo com a faixa etária e gênero

\begin{tabular}{lccc}
\hline \multirow{2}{*}{ Faixa etária } & \multicolumn{2}{c}{ Gênero } & Total \\
& Masculino & Feminino & \\
\hline $1-4$ anos & 3 & 0 & 3 \\
$4 \vdash 8$ anos & 6 & 2 & 8 \\
$8 \vdash 13$ anos & 10 & 5 & 15 \\
\hline Total & 19 & 7 & 26 \\
\hline
\end{tabular}

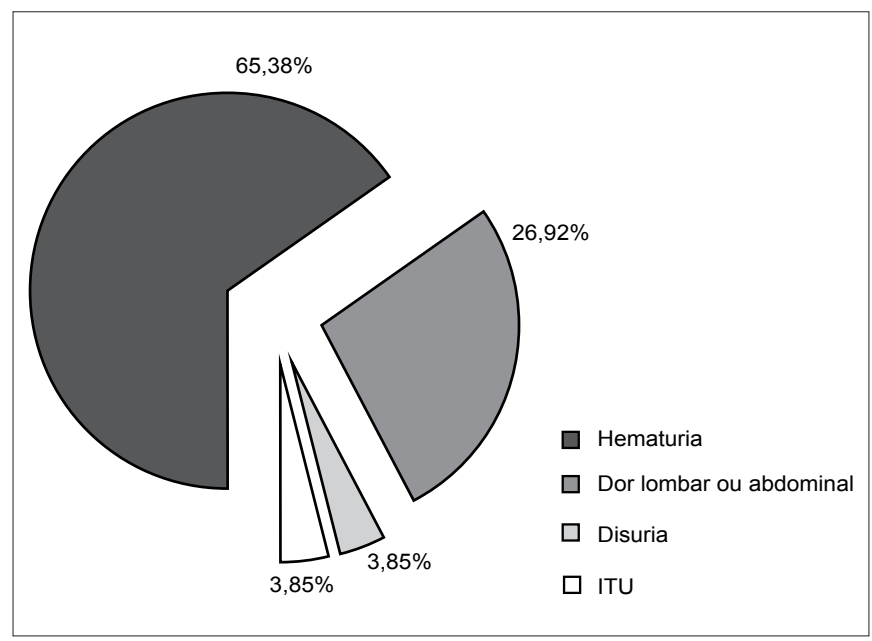

Gráfico 1 - Distribuição dos pacientes de acordo com apresentação clínica inicial

Tabela 2 - Mediana e semi-amplitude interquatílica dos valores de creatinina, cálcio, sódio, potássio séricos e urinários e das relações $\mathrm{Ca} / \mathrm{Cr}, \mathrm{K} / \mathrm{Cr}, \mathrm{Na} / \mathrm{Cr}$ e $\mathrm{Na} / \mathrm{K}$ urinários e respectivo resultado do teste estatístico nos dois momentos

\begin{tabular}{lccc}
\hline & Pré-tratamento & Pós-tratamento & $p$ \\
\hline Creatinina $(\mathrm{mg} / \mathrm{dL})$ & $0,60 \pm 0,05$ & $0,50 \pm 0,15$ & $>0,05$ \\
Cálcio $(\mathrm{mg} / \mathrm{dL})$ & $9,7 \pm 0,2$ & $9,5 \pm 0,4$ & $>0,05$ \\
Sódio $(\mathrm{mEq} / \mathrm{L})$ & $143,0 \pm 1,0$ & $142,5 \pm 1,5$ & $>0,05$ \\
Potássio $(\mathrm{mEq} / \mathrm{L})$ & $4,4 \pm 0,4$ & $4,2 \pm 0,3$ & $>0,05$ \\
Cálcio $(\mathrm{mg} / 24 \mathrm{~h})$ & $161,5 \pm 43,3$ & $115,1 \pm 37,3$ & $<0,01$ \\
Potássio $(\mathrm{mEq} / 24 \mathrm{~h})$ & $24,3 \pm 15,3$ & $39,7 \pm 15,5$ & $<0,001$ \\
Sódio $(\mathrm{mEq} / 24 \mathrm{~h})$ & $117,1 \pm 41,8$ & $116,2 \pm 39,0$ & $>0,05$ \\
$\mathrm{Ca} / \mathrm{Cr}$ & $0,28 \pm 0,09$ & $0,22 \pm 0,07$ & $<0,01$ \\
$\mathrm{~K} / \mathrm{Cr}$ & $0,04 \pm 0,01$ & $0,08 \pm 0,03$ & $<0,01$ \\
$\mathrm{Na} / \mathrm{Cr}$ & $0,22 \pm 0,06$ & $0,22 \pm 0,05$ & $>0,05$ \\
$\mathrm{Na} / \mathrm{K}$ & $5,54 \pm 1,78$ & $3,66 \pm 1,40$ & $<0,005$ \\
\hline
\end{tabular}




\section{Discussão}

A sintomatologia da hipercalciúria é variável, podendo se manifestar com diferentes sinais e sintomas clínicos, sendo os mais comuns: hematúria (micro ou macroscópica), dor abdominal incaracterística e disúria. Pode também se apresentar com enurese, urgência, incontinência urinária e dor suprapúbica ${ }^{(2,4,21,22)}$.

Nesse estudo, sinais e sintomas de hematúria $(65,4 \%)$ e dor abdominal ou lombar (27\%) foram os mais prevalentes no início da doença, estando em concordância com a literatura $^{(2,23)}$. Tais achados reforçam a necessidade de investigar alterações metabólicas em toda criança que apresentar esta sintomatologia. A hipercalciúria idiopática é a principal alteração metabólica responsável pela formação de cálculos em adultos ${ }^{(24,25)}$ e em crianças $^{(26,27)}$.

O tratamento da hipercalciúria idiopática inclui: adequação da dieta em relação ao sódio e à proteína, aumento da ingesta hídrica, uso de citrato de potássio e diuréticos tiazídicos ${ }^{(11,27,28)}$. $\mathrm{O}$ ajuste na ingestão protéica está indicado quando o consumo é aumentado, o que acarreta acidose e maior excreção urinária de cálcio ${ }^{(16)}$. A restrição de alimentos ricos em cálcio, preconizada anteriormente em relação aos leites e derivados ${ }^{(18)}$, não mais se justifica frente ao melhor conhecimento da fisiopatologia da doença e à constatação da associação do pior prognóstico da doença óssea hipercalciúrica com a restrição de cálcio ${ }^{(3,29,30)}$.

Os efeitos dos diuréticos tiazídicos sobre a hipercalciúria idiopática são decorrentes do aumento da reabsorção proximal de cálcio, pela contração do volume extracelular ${ }^{(31)}$. O citrato de potássio é utilizado na litíase, por ser um quelante que se liga ao cálcio ionizado disponível na urina, inibindo o crescimento e a agregação de cristais urinários com oxalato de cálcio. Esta substância promove a alcalinização da urina, além de repor o citrato nos pacientes com hipocitratúria ${ }^{(19)}$. É também utilizado como medicamento de primeira escolha em crianças com hipercalciúria idiopática ${ }^{(2)}$.

Estudo em adulto mostrou que o aumento na excreção de potássio eleva a reabsorção tubular renal de cálcio, sendo atribuída esta propriedade terapêutica ao citrato de potássio ${ }^{(18)}$. O modo pelo qual o aumento na excreção urinária de potássio reduz a excreção renal de cálcio ainda não está totalmente esclarecido. Acredita-se que o potássio promova a retenção renal de cálcio diretamente ${ }^{(16,18,32)}$. Outro efeito do potássio é a retenção de fósforo, o qual inibe o calcitriol e, subseqüentemente, a absorção intestinal de cálcio e, finalmente, a calciúria, sendo este um mecanismo indireto ${ }^{(33)}$.

Quando se avaliou isoladamente a excreção urinária de cada eletrólito em todo o grupo, foi constatada redução significativa na excreção do cálcio após uso de citrato de potássio e, ao mesmo tempo, aumento significativo na excreção urinária de potássio. Tais resultados estão de acordo com a literatura ${ }^{(18,34)}$. O valor da excreção urinária de potássio no presente estudo, no momento pré-citrato $(0,95 \pm 0,36 \mathrm{mEq} /$ $\mathrm{kg} / \mathrm{dia}$ ) foi semelhante ao encontrado por outros autores em crianças hipercalciúricas ${ }^{(35)}$. Esses autores encontraram valores da excreção de potássio de $1,1 \pm 1,3 \mathrm{mEq} / \mathrm{kg} / \mathrm{dia}$.

O comportamento da relação de cálcio/creatinina e potássio/creatinina foi semelhante ao notado por outros autores, com redução da relação cálcio/creatinina e aumento da relação potássio/creatinina após a ingestão de citrato de potássio ${ }^{(18)}$. Não houve variação significativa do sódio, o que mostra que o uso oral de citrato de potássio não aumentou a natriúria.

A relação $\mathrm{Na} / \mathrm{K}$, nos pacientes hipercalciúricos na fase pré-tratamento, se mostrou maior $(5,68 \pm 2,73)$ do que a observada na literatura ${ }^{(18,35)}: 4,73 \pm 2,28 ; 4,2 \pm 3,9$, sugerindo ocorrer maior ingestão de sódio pelos pacientes do presente trabalho. $\mathrm{O}$ valor considerado normal da relação $\mathrm{Na} / \mathrm{K}$ urinários é <2; no entanto, nas crianças com hipercalciúria, em geral, este valor é maior que $4,5^{(18,35)}$. A alta ingestão de sódio em crianças hipercalciúricas brasileiras foi observada em outro estudo, no qual se comparou a ingestão do sódio com a recomendada pela RDA (Recommended Dietary Allowance $)^{(7,36)}$. Após o uso de citrato de potássio, houve redução da relação $\mathrm{Na} / \mathrm{K}$ devido ao aumento na excreção de potássio, já que a diminuição na excreção de sódio não chegou a ser significativa.

Os resultados indicam a necessidade de recomendação dietética rica em potássio como medida básica para os pacientes com hipercalciúria idiopática. O emprego de citrato de potássio em crianças hipercalciúricas em dieta habitual promove elevação da excreção urinária de potássio e diminuição da excreção urinária de cálcio, constituindo-se em importante recurso terapêutico na hipercalciúria idiopática. 


\section{Referências bibliográficas}

1. Perrone HC, Sigulen D, Toporovski J, Schor N. Normatização da excreção urinária de cálcio e ácido úrico em crianças. J Bras Nefrol 1990;12:23-8.

2. Penido MG, Diniz JS, Moreira ML, Tupinambá AL, França A, Andrade BH et al. Hipercalciúria idiopática: apresentação de 471 casos. J Pediatr (Rio J) 2001;77:101-4.

3. Langman CB. Disorders of phosphorus, calcium and vitamin D. In: Avner ED, Harmon WE, Niaudet P. Pediatric Nephiology. $5^{\text {th }}$ ed. Baltmore: Lippincott; 2004. p.237-54.

4. Alon US, Berenbom A. Idiopathic hypercalciuria of childhood: 4- to 11-year outcome. Pediatr Nephrol 2000;14:1011-5.

5. Perrone HC, Ajzen H, Toporovski J, Schor N. Metabolic disturbance as a cause of recurrent hematuria in children. Kidney Int 1991;39:707-10.

6. Diniz JS, Silva JM, Lima EM, Cardoso LS, Moreira ML, Silva AC et al. 30 anos de experiência em nefrologia pediátrica: um estudo descritivo. J Bras Nefrol 2005;27:201-6.

7. Andrade AS, Jalles LM, Lopes MF, Oliveira CC, Brito TN, Pedrosa LF. Proteína, cálcio e sódio na dieta habitual de crianças e adolescentes do Rio Grande do Norte com nefrolitíase. J Bras Nefrol 2004;26:76-83.

8. Stapleton FB. Hematuria associated with hypercalciuria and hyperuricosuria: a practical approach. Pediatr Nephrol 1994;8:756-61.

9. Penido MGMG. Alterações ósseas na hipercalciúria idiopática em crianças e adolescentes [tese de doutorado]. Belo Horizonte (MG): UFMG; 2000.

10. Martini LA, Heilberg IP, Schor N. Papel dos fatores dietéticos na litogênese. In: Schor N, Heilberg IP. Calculose renal: fisiopatologia, diagnóstico, tratamento. $1^{\text {a }}$ ed. São Paulo: Sarvier, 1995; p.31-5.

11. Muldowney FP, Freaney R, Moloney MF. Importance of dietary sodium in the hypercalciuria syndrome. Kidney Int 1982;22:292-6.

12. Breslau NA, McGuire JL, Zerwekh JE, Pak CY. The role of dietary sodium on renal excretion and intestinal absorption of calcium and on vitamin D metabolism. J Clin Endocrinol Metab 1982;55:369-73.

13. O'Brien KO, Abrams SA, Stuff JE, Liang LK, Welch TR. Variables related to urinary calcium excretion in young girls. J Pediatr Gastroenterol Nutr 1996;23:8-12.

14. Aladjem M, Barr J, Lahat E, Bistritzer T. Renal and absorptive hypercalciuria: a metabolic disturbance with varying and interchanging modes of expression. Pediatrics 1996;97:216-9.

15. Cirillo M, Laurenzi M, Panarelli W, Stamler J. Urinary sodium to potassium ratio and urinary stone disease. Kidney Int 1994;46:1133-9.

16. Lemann J Jr, Pleuss JA, Gray RW, Hoffmann RG. Potassium administration reduces and potassium deprivation increases urinary calcium excretion in healthy adults [corrected]. Kidney Int 1991;39:973-83.

17. Rodriguez-Soriano J, Ubetagoyena M, Vallo A. Renal potassium excretion is reduced in children with idiopathic hypercalciuria. Miner Electrolyte Metab 1991;17:357-61.

18. Osório AV, Alon US. The relationship between urinary calcium, sodium, and potassium excretion and the role of potassium in treating idiopathic hypercalciuria. Pediatrics 1997;100:675-81.
19. Perrone HC, dos Santos DR, Santos MV, Pinheiro ME, Toporovski J, Schor N. Estudo metabólico da nefrolitíase na infância: avaliação em cinqüenta crianças litiásicas. J Pediatr (Rio J) 1993;69:36-41.

20. Norman GR, Streiner DL. Biostatistics - the bare essentials. $2^{\text {nd }}$ ed. St Louis: Mosby; 2000.

21. Alon U, Warady BA, Hellerstein S. Hypercalciuria in the frequency-dysuria syndrome of childhood. J Pediatr 1990;116:103-5.

22. Polito C, La Manna A, Cioce F, Villani J, Nappi B, Di Toro R. Clinical presentation and natural course of idiopathic hypercalciuria in children. Pediatr Nephrol 2000;15:211-4.

23. Stapleton FB. Idiopathic hypercalciuria: association with isolated hematuria and risk for urolithiasis in children. Kidney Int 1990;37:807-11.

24. Coe FL, Favus MJ, Crockett T, Strauss AL, Parks JH, Porat A et al. Effects of low-calcium diet on urine calcium excretion, parathyroid function and serum $1,25(\mathrm{OH}) 2 \mathrm{D} 3$ levels in patients with idiopathic hypercalciuria and in norma subject. Am J Med 1982;72:25-32.

25. Stapleton FB, Roy S $3^{\text {rd }}$, Noe HN, Jerkins G. Hypercalciuria in children with hematuria. N Engl J Med 1984;310:1345-8.

26. Perrone HC, Schor N. Litíase: aspectos clínicos. In: Schor N, Srougi M. Nefrologia e urologia clínica. $1^{\mathrm{a}}$ ed. São Paulo: Sarvier; 1988. p. 370-3.

27. Pak CY. Etiology and treatment of urolithiasis. Am J Kidney Dis 1991;18:624-37.

28. Moore ES, Coe FL, McMann BJ, Favus MJ. Idiopathic hypercalciuria in children prevalence and metabolic characteristics. J Pediatr 1978;92:906-10.

29. Cervera A, Corral MJ, Gómez Campadera FJ, De Lecea AM, Luque A, Lopez Gómez JM. Idiopathic hypercalciuria in children: classification, clinical manifestations and outcome. Acta Pediatr Scand 1987;76:271-8.

30. Weisinger JR. New insights into the pathogenesis of idiopathic hypercalciuria the role of bone. Kidney Int 1996;49:1507-18.

31. Schor N, Heilberg IP. Tratamento clínico da litíase urinária. In: Schor N, Heilberg

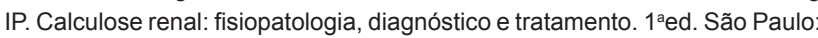
Sarvier; 1995. p. 205-11.

32. Jones JW, Sebastian A, Hulter HN, Schambelan M, Sutton JM, Biglieri EG. Systemic and renal acid-base effects of chronic dietary potassium depletion in humans. Kidney Int 1982;21:402-10.

33. Jaeger P, Bonjour JP, Kalmark B, Stanton B, Kirk RG, Duplinsky T et al. Influence of acute potassium loading on renal phosphate transport in the rat kidney. Am J Physiol 1983;245:F601-5.

34. Calò L, Borsatti A, Favaro S, Rabinowitz L. Kaliuresis in normal subjects following oral potassium citrate intake without increased plasma potassium concentration. Nephron 1995;69:253-8.

35. Polito C, La Manna A, Maiello R, Nappi B, Siciliano MC, Di Domenico MR et al. Urinary sodium and potassium excretion in idiopathic hypercalciuria of children. Nephron 2002;91:7-12.

36. Penido MG, Diniz JS, Guimarães MM, Cardoso RB, Souto MF, Penido MG. Excreção urinária de cálcio, ácido úrico e citrato em crianças e adolescentes sadios. J Pediatr (Rio J) 2002;78:153-60. 